\title{
A MultidisciplinARY ELECTROCHEMICAL ENGINEERING LABORATORY COURSE
}

\author{
Daniel T. Schwartz \\ Department of Chemical Engineering \\ Box 351750 \\ University of Washington \\ Seattle, WA 98195-1750
}

Roughly $5 \%$ of the U.S. gross national product is spent on value-added electrochemical processes or value-degrading electrochemical corrosion. ${ }^{1}$ This sizable economic impact suggests that many, if not most, engineers and scientists will grapple with electrochemical issues during their careers. The ways in which engineers and scientists interact with electrochemical processes is sometimes obvious. For example, electrodeposition may be used for coating a substrate, corrosion may be a process design constraint, or electroanalytical chemistry may be used to detect a contaminant. On the other hand, the role of electrochemistry is often subtle, yet important. For example, cell membrane potentials are dictated by electrolytic processes, electrochromic smart window technology depends on ion conducting materials, charge transfer reactions impact the efficacy of soil dewatering with electroosmosis, and more. Despite the ubiquitous nature of electrochemical phenomena, few students in the U.S. have an opportunity to become well-founded in electrochemical science and technology. In fact, the status of electrochemical education has changed little since a 1986 National Research Council report noted, ${ }^{1}$

\begin{abstract}
"Most of the chemistry and chemical engineering departments offer no formal instruction or research in corrosion. Fewer than 20 percent of the chemical engineering departments provide training in electrochemical synthesis and energy conversion, either through course work or research. In addition, over the past 10 to 20 years there has been a gradual disappearance of electrochemical coverage in most physical chemistry courses."
\end{abstract}

In short, a sizable fraction of all practicing engineers and scientists will use electrochemical principles in their work, though most will only receive piece-meal training through on-the-job experience. It is accurate to say that the transmission of modern electrochemical insight to students has lagged far behind the pace of cutting-edge research advances.

For the past six years, students at the University of Washington have had the opportunity to learn about electrochemical science and technology, initially in a lecture-oriented advanced undergraduate course, and more recently as a laboratory-oriented course. The objectives of this course have always been to develop in students a broadly based understanding of electrochemical processes, to effectively integrate cutting-edge research advances into the undergraduate curriculum, and to support efforts within chemical engineering and across campus to have students work on industry-inspired and multidisciplinary design projects. The transition from lecture to laboratory course was driven by a desire to have the multidisciplinary students learn and retain more, and also to help them develop insight and intuition about electrochemical 
processes. An experiential learning process that combines lectures and hands-on experiments has proven to be effective at accomplishing these objectives. The course is called Electrochemical Engineering.

The degree level and disciplinary affiliation of students in Electrochemical Engineering has been somewhat broader than the author typically finds in other engineering course. Approximately half of the students who have attended the course during the past 6 years have been undergraduate students ( 36 total), and the other half have been graduate or nonmatriculated students from local industry (40 total). From these numbers, one sees that typical enrollment averages about 13 students per quarter. Chemical engineers make up about $80 \%$ of the course enrollment, whereas students from other engineering departments, the College of Arts and Sciences, the College of Forestry, and industry comprise the remaining enrollment. The specific departmental affiliations of students have included engineers from the bio, chemical, civil, materials, and interdisciplinary engineering programs, together with students from forest resources, chemistry, biochemistry, and undeclared Arts and Sciences majors.

The original lecture-oriented Electrochemical Engineering course was fairly mathematical, drawing its material heavily from a presumed background in engineering science and chemistry courses. Because of the wide mix of students with their varied backgrounds in mathematics and chemistry, in-class demonstrations were added to the lecture material beginning in the second year of the course. These in-class demonstrations illustrated basic points in cell thermodynamics, charge transfer kinetics, and convective mass transfer. The student evaluations broadly indicated that the experimental demonstrations were the most effective learning approach in the course. This student feedback provided the impetus to seek the support needed to establish an experiential learning environment where a laboratory supplanted, for the most part, classroom and homework activities. To date, the laboratory has involved a total investment of approximately $\$ 126,000$, three quarters of which has gone into equipping experimental stations. Support for the laboratory has come mostly from private industry and a private educational foundation (60\% of total), but the early and generous commitment of university and departmental resources was central to establishing the laboratory. Operating costs for the laboratory are modest, except for the salary provided to the professor to teach the course. No teaching assistance is formally provided, though graduate students from the author's laboratory often help with preparation of electrolytes and such.

Electrochemical Engineering has evolved to include three distinct sets of experiments. A four week experimental module called electrochemical sensing starts the quarter off. In this experiment, students explore the foundations of current-potential response in electrolytic systems. Next, the students perform a two week electrochemical technology module with a variety of experiments drawn largely from the author's graduate research laboratories. Finally, the students perform a two week technology-oriented experimental module either designed by student teams to integrate with their senior design or graduate research projects, or they can perform another of the technology experiments drawn from the author's graduate research program. To the best of my knowledge, this course is quite unique in its mission and approach, 
being neither an electroanalytical chemistry laboratory nor a lecture-oriented electrochemical engineering course. The course is taught in a ten week quarter. Eighty minutes of lecture are presented weekly. Grades are based on 3 written reports (70\%), a midterm exam (20\%) and an oral report $(10 \%)$.

Having just provided the context, motivation, resources, and overall organization for this Electrochemical Engineering laboratory, it is perhaps worthwhile to describe some assessment outcomes before detailing the individual experiments. During the initial four year period when the course was lecture-oriented, the response of students to the course evaluation question, "The amount you learned in the course was: Excellent (5), Very Good (4), Good (3), Fair (2), Poor (1), Very Poor (0)" averaged 3.2, and the response to the question, "The course as a whole was: Excellent (5), Very Good (4), Good (3), Fair (2), Poor (1), Very Poor (0)" averaged 3.6. Only one set of student assessments have been received since the course converted to a laboratoryoriented learning experience (1998 assessments have not been returned as of press time). Nonetheless, from the student perspective, the laboratory course appears to be more successful than the previous lecture format. In most assessment categories, student ratings for the laboratory have exceeded the highest ratings received in any of the four years of lecturing. In response to the first and second question quoted above, the laboratory-oriented course received scores of 3.9 and 4.3, respectively. The 4.3 score for "Course as a whole" placed the Electrochemical Engineering laboratory in the top quintile for College of Engineering courses at the University of Washington. Aside from student input, the main alternative assessment tool is the author's subjective opinion regarding how the students tackle the midterm exam and the knowledge displayed in laboratory reports; the amount of learning seems to have improved dramatically.

The laboratory modules outlined below are designed to reinforce the fundamentals of electrochemical engineering by having students work on electrochemical experiments that employ thermodynamics, reaction kinetics, and mass transfer via convection, diffusion, and migration. All of the students, working as two person teams, perform the Electrochemical Sensing module as their first experiment. The electrochemical sensing module serves as an introduction and overview of potentiometric and amperometric measurements. The student teams measure the composition and physical properties of simple electrolytes (ferrous and ferric sulfate in acidic media) using a variety of electroanalytical tools. These experiments use both rotating and stationary electrodes. The students measure quantities and apply theories that describe the influence of solution thermodynamics, charge transfer kinetics, and steady and unsteady mass transfer rates on the potentiometric and amperometric response of an electrode placed in an electrolyte. Moreover, they also are exposed to important fundamental and practical issues in electrochemistry (e.g. the role of activity coefficients in potentiometric measurements and the need for clean, reproducible surfaces for amperometric techniques). This experiment lays the foundation for more application-oriented projects; electrochemical sensing is the type of experiment students might get in an electroanalytical laboratory course. 
After completing the electrochemical sensing experiments, students then proceed (as teams of two, ideally) to a selection of electrochemical technology experiments drawn from the author's research laboratories. Each student team selects one of the experiments described below, based on their interest. Teams are often reformulated to match students who are interested in performing the same experiments. The electrochemical technology experiments are outlined below.

Electrodeposition of Nanostructured Alloys. One of the major research thrusts in the author's graduate research program is the electrodeposition of nanostructured alloys. ${ }^{2,3}$ In this laboratory experiment, the students electroplate $\mathrm{NiFe}$ alloys using a rotating ring-disk electrode system under steady and oscillating rotation rate conditions. Electroplated $\mathrm{NiFe}$ is the soft magnetic material used in computer disk-drive assemblies. Plating under steady flow conditions results in homogeneous alloy films, but oscillating flow conditions result in a composition modulated alloy (a layered sandwich structure) since the alloy composition is highly dependent on the strength of agitation. ${ }^{2,3}$ The composition of the alloy is determined by measuring (at the ring of the ring-disk electrode) the ferrous ions liberated from the deposit while it is anodically etched. Students have successfully made and characterized nanostructures with layers as thin as $12 \mathrm{~nm}$. This experiment illustrates the importance of equilibrium considerations when there are simultaneous reactions and the role of convective mass transfer in electrochemical systems, while simultaneously exposing students to the excitement of nanotechnology.

Environmentally Benign Ion Exchange. The largest single graduate research effort currently ongoing in the author's graduate laboratories involves studies of electrochemically switched ion exchange processes as alternatives to traditional ion exchange. ${ }^{4}$ In traditional ion exchange, chemical potential is normally modulated (via changes in solution concentration) to cause ions to load and elute from the exchange matrix. In this laboratory, students deposit and characterize electrodes derivatized with a nickel hexacyanoferrate (NiHCF) ion exchange material. By changing the potential on the electrode, they can modulate the charge density on the exchange matrix, thereby causing cations to load or elute to maintain charge neutrality in the matrix. Students qualitatively study the selectivity of the matrix for cesium cations over sodium using cyclic voltammetry, and they quantitatively determine ion diffusivities in the matrix. The recent acquisition of a quartz crystal nanobalance for the laboratory means that next year students will be able to weight the ions loaded into the film to quantitatively determine ion affinities for the matrix. This experiment reinforces basic thermodynamic concepts, determination of physical properties, and exposes the students to emerging separation methods.

Electrochromic Conducting Polymers. As part of our graduate research effort in nanostructured materials, we have dabbled in the electrodeposition and characterization of conducting polymer materials. In this laboratory, the students study the redox and optical behavior of electronically conducting polyaniline thin films that are deposited on transparent fluorine-doped tin oxide substrates. Polyaniline is an electrochromic material that changes color as the film changes oxidation state; ${ }^{5}$ it serves as a model for electrochromic display materials for video screens. Students use premade polyaniline thin films of differing thicknesses (the actual electrodeposition 
of polyaniline requires handling somewhat hazardous materials, so the instructor does that in advance). By performing cyclic voltammetry on the films, they are able to determine the film thickness (via redox charge) and also map-out the potential regimes where the film exhibits various colors. The changes in film color are analyzed quantitatively using UV-Vis spectroscopy to determine the optical absorption spectrum of polyaniline as a function of potential. In addition, the dynamics of electrochemical film switching (transition from transparent to colored) is analyzed to assess polyaniline's suitability as a video display material. These experiments reinforce basic electrochemical concepts like Faraday's Law, charge transfer dynamics and molecular charge balances. In addition, they also show how the electronic and optical properties of molecules can be engineered by modifying their charge density and bonding traits.

Characteristics of Rechargeable Batteries. Batteries are widely encountered electrochemical devices and, it seems, students often have questions about how and why they work the way they do. In this laboratory, students study the charge and discharge characteristics of commercial AAA NiCd batteries. Cell thermodynamic studies are performed by measuring the equilibrium potential of the battery at three different temperatures. From this data students determine the Gibbs free energy, enthalpy, and entropy of reaction for the overall cell reaction $2 \mathrm{NiOOH}+\mathrm{Cd}+$ $2 \mathrm{H}_{2} \mathrm{O} \varnothing 2 \mathrm{Ni}(\mathrm{OH})_{2}+\mathrm{Cd}(\mathrm{OH})_{2}$. Constant rate discharge and recharge curves allow the students to determine the energy and power density, as well as the energy efficiency. Not only does this experiment allow the students to answer, for themselves, the reasons why a battery works the way it does, but it also lets them learn to manipulate thermophysical data and understand the full interplay of kinetic and ohmic loss mechanisms in galvanic devices.

The final two week experimental module allows students to select another of the technology experiment described above, or they can develop a special project related to their senior design or graduate project. The special projects are developed through consultation with the Electrochemical Engineering instructor and the instructor for the design/graduate project. In 1998, three different special projects were developed, two were related to a chemical milling process employed by Boeing, and one was a project related to a college-wide multidisciplinary design project to make a fuel cell powered locomotive. Boeing uses chemical etching to remove excess aluminum from aircraft components-the processes is essentially controlled corrosion. One student group looked at etch rate and solution potential dependence as a function of temperature, whereas the other student group assessed whether electroanalytical techniques and UV-Vis spectroscopy could be used as in-situ sensors to characterize the chemical etching solution (for control purposes). The student team studying fuel cell materials tried to characterize the ionic conductivity of a Nafion membrane used as the proton conducting electrolyte in a hydrogen/oxygen polymer electrolyte membrane fuel cell.

The preceding text makes many of the lessons that have been learned fairly transparent- the value of experiential learning when working across disciplines, the expense of experiential learning, the ease with which research and education can be merged in a topical laboratory course, the role of such a course to enhance and meld design across the curriculum - but one point is not as obvious. The total monetary support necessary to develop this course (about 
$\$ 126,000)$ was less than $10 \%$ of the Private, University, and Federal funding that supported the graduate level research that forms the underpinning of this undergraduate course. Research funding during the past six years has directly impacted 7 Ph.D. and 2 M.S. students. For an additional $10 \%$ investment beyond graduate research support, one can develop a course like Electrochemical Engineering that has the potential to impact 16 students every quarter the course is taught. Thus, putting resources into the integration of research and teaching appears to be a sound investment for leveraging the value of research progress.

Acknowledgment-Financial support has been provided by the Camille and Henry Dreyfus Foundation, IBM Corporation, Electrochemical Technology Corporation, and the University of Washington. Professor Bruce A. Finlayson's encouragement, support, and promotion of this course is greatly appreciated.

[1] "New horizons in electrochemical science and technology," Publication NMAB 438-1, National Academy Press, Washington, D.C. (1986).

[2] S.D. Leith and D.T. Schwartz, J. Electrochem. Soc., 143, 873 (1996).

[3] J.A. Medina and D.T. Schwartz, Electrochim. Acta 42, 2679 (1997).

[4] M.A. Lilga, R.J. Orth, J.H. Sukamto, S.M. Haight, and D.T. Schwartz, Sep. Purif. Tech., 11 147 (1997).

[5] W-S. Huang, B.D. Humphrey, and A.G. Macdiarmid, J. Chem. Soc. Faraday Trans. I, 82, 2385 (1986).

DANIEL T. SCHWARTZ is Associate Professor of Chemical Engineering at the University of Washington. He joined the University of Washington in 1991. 